\title{
SIMULATION OF LINE ARRAY SOUND SOURCE
}

This paper is focused on the simulation of line array source that consists of eight separate loudspeaker enclosures of the same type having some directional characteristics influenced by the position within the line array. The influence on these characteristics caused by the different positions of enclosures in the array was tested. The characteristics of the line array source were measured in the anechoic chamber. The comparison between the results of the simulation of ideal and real sources is shown.

Keywords: Sound source, acoustic signal, acoustic simulation, directional characteristics.

\section{Introduction}

Sound systems are nowadays increasingly confronted with the requirement of high fidelity sound reproduction. This is closely related to the spectral composition of reproduced acoustic signal. Thus, the reproduced signal should contain the same spectral components as the original and have the least possible distortion (linear or nonlinear). However, high fidelity also increasingly depends on the transfer of information about the original sound source location. It means the direction and distance of the original source related to the listener. For example, this information is very important in the area of teleconferencing technology, but also in the area of arts or entertainment. Among other methods of multichannel sound reproduction systems there is wave field synthesis (WFS) [1]. WFS is a modern method of sound reproduction allowing the true reproduction of sound field including the spatial information. This method utilizing almost exclusively horizontally installed line array sources for the reproduction of acoustic signal.

It is very useful to simulate the acoustic field generated by single sound source or even by more sources. The final image can display the interaction of the sources in areas where they are summed together according to the phase and amplitude relations between them. Therefore, we can get the picture of sound pressure level in the area in front of the loudspeaker array.

The method of ideal sound source simulation is presented in [2]. In our work, we take measured directional characteristics of real sources into account, including diffraction.

The line array sound source described in this paper was designed as the sound source for the use with the WFS. The system consists of eight loudspeaker enclosures, each of the same construction and with 3-inch speaker inside.

The paper presents the process of directional characteristics measurement and subsequent application of the measured data to simulate directional behavior of the real sources. All the simulations were done in Matlab.

\section{Ideal sound source simulation}

The essential assumption for all of the presented simulations is that all sources are placed in the free field. Therefore, reflections from the walls or other barriers are not considered [3]. Moreover, the simulations and measurement were done only in the horizontal plane. One of the possible ways of sound field description is utilizing the velocity potential $\Phi[3]$ and [4]. It is a scalar function and acoustic (particle) velocity $v=\operatorname{grad} \Phi$ can be calculated as [5]. An advantage of description with velocity potential is the scalar addition of individual sources contributions as described

$$
\Phi=\sum_{i=1}^{n} \Phi_{i}
$$

It means that the total velocity potential in every point of the space is the sum of potentials induced of all the sources. Then acoustic pressure $p$ of harmonic wave with angular frequency $\omega$ can be written as

$$
p=-j \omega \rho_{0} \Phi
$$

where $\rho_{0}$ is medium density (air density in our case).

Basic source used in acoustic simulations is a point source [6]. Its main property is frequency-independent omnidirectional polar pattern. Velocity potential of the $i$-th point source at the point $\mathrm{X}$ in distance of $r_{i}$ from the source can be calculated as [3] and [4]

$$
\Phi_{i}=A_{i} \frac{e^{-j k r_{i}}}{r_{i}}
$$

\footnotetext{
* David Kurc, Jiri Schimmel

Department of Telecommunications, FEEC, Brno University of Technology, Czech Republic, E-mail: david.kurc@phd.feec.vutbr.cz
} 
where $A_{i}$ is an amplitude of the source and $k$ is the wave number ( $k=\omega / c_{0}$, where $c_{0}$ is speed of sound). However, the real sources (loudspeakers) can be considered as the point sources only in the situations when the wavelength of emitted sound wave is much greater than physical dimensions of the source [6]. Nevertheless, only very low frequencies satisfy this requirement - more precisely, the frequencies meeting condition $k R<1$, where $R$ is the radius of speaker diaphragm. In other cases it is more accurate to utilize vibrating circular piston sources in the simulations [4]. It is considered to be located on the wall surface, which is perfectly rigid, flat and infinitely large. Due to this assumption, there is no need to consider the phenomena associated with diffraction on the edges [4]. However, the properties of this kind of source are more similar to the real loudspeakers with circular diaphragm, especially in directional characteristics. Velocity potential of the $i$-th vibrating circu- lar piston source at the point $\mathrm{X}$ in distance of $r_{i}$ from the source can be calculated as [4]

$$
\Phi_{i}=-A_{i} \frac{e^{-j k r_{i}}}{r_{i}} \pi R_{i}^{2} \frac{2 J_{1}\left(k R_{i} \sin \vartheta_{i}\right)}{k R_{i} \sin \vartheta_{i}}
$$

where $R_{i}$ is the radius of $i$-th circular piston source, is the Bessel function of the first kind and $\vartheta_{i}$ is the angle between the acoustic axis of the loudspeaker and the line connecting the speaker reference point and calculated point $\mathrm{X}$. For example, the loudspeakers used for our simulations have the diaphragm radius of $3.81 \mathrm{~cm}$. Thus, eq. (3) can be used for the simulations of frequencies below $1.42 \mathrm{kHz}$, while eq. (4) have to be used for higher frequencies.

The comparison example of these kinds of sources with the frequency of $4 \mathrm{kHz}$ can be seen in Fig. 1. As can be seen in the
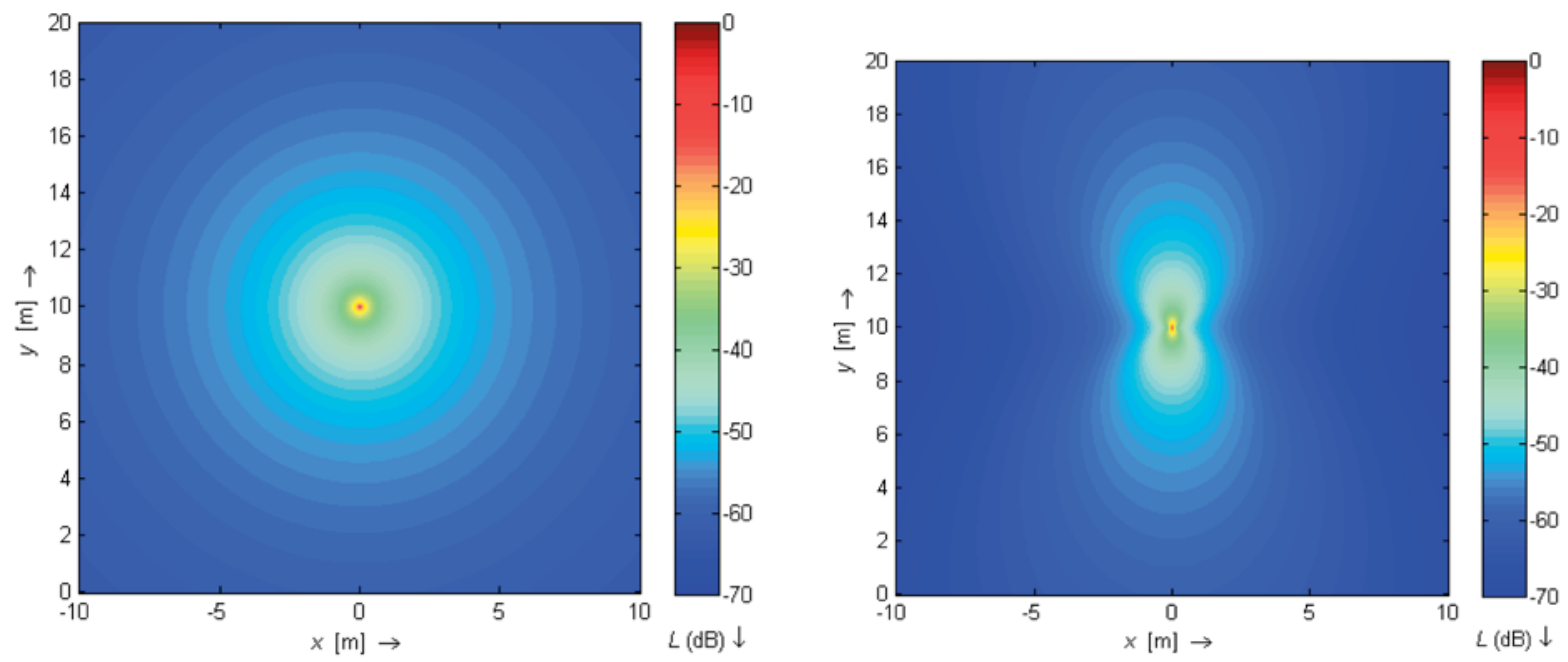

Fig. 1 The comparison of point source (left) and circular piston source (right) at the frequency of $4 \mathrm{kHz}$. Diaphragm radius is $3.81 \mathrm{~cm}$
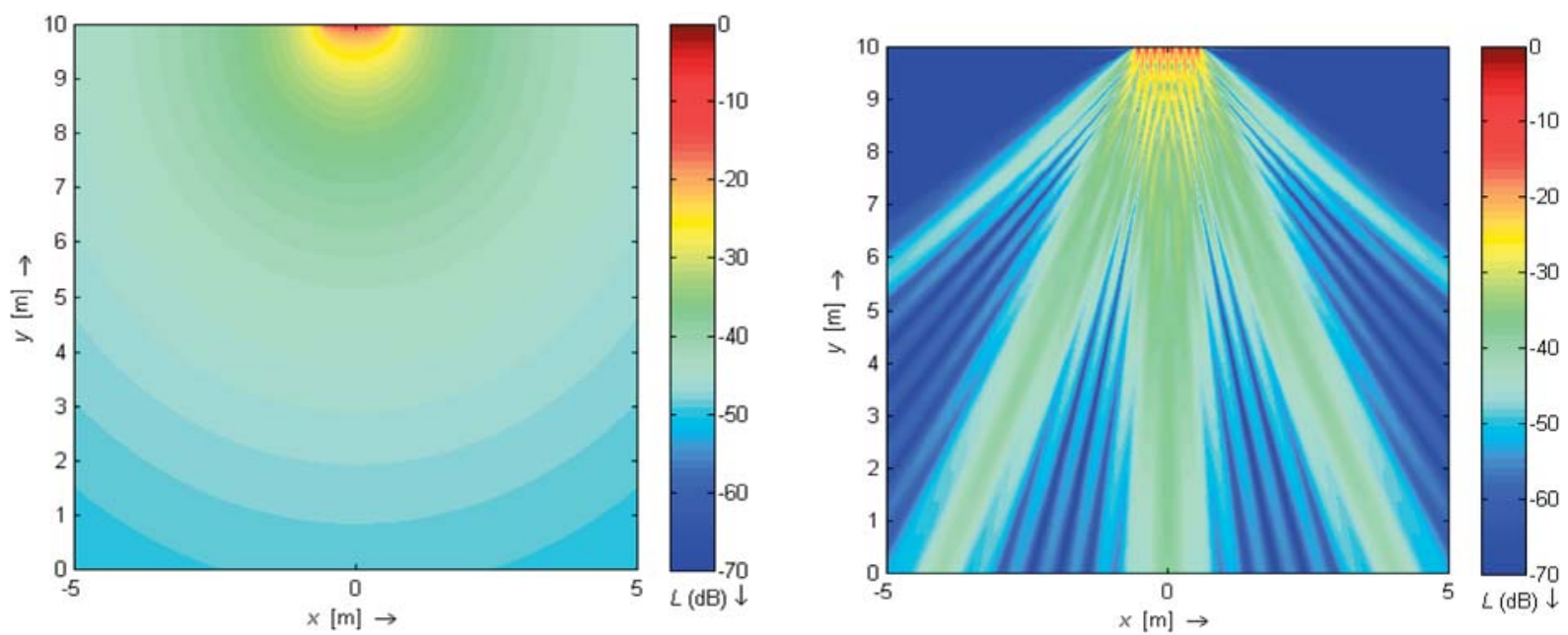

Fig. 2 The line array source simulation with eight individual sources spaced $15 \mathrm{~cm}$ at the frequency of $100 \mathrm{~Hz}$ (left) and at the frequency of $6 \mathrm{kHz}$ (right). Diaphragm radius is $3.81 \mathrm{~cm}$ 
figures, the main difference between these sources is in their directivity. Polar pattern of a point source is the same at all frequencies while the polar pattern of a circular piston source gets narrower with increasing frequency.

An important reason for simulation of sound pressure distribution is the visualization of interactions between two and more sources. Two major factors are crucial in the process of source summing. They are the difference in amplitude and the difference in phase of the sources [6] and [7]. The resulting picture of the interaction of the line array source containing eight loudspeaker enclosures at low frequency and high frequency is shown in Fig. 2. The point source model was used for low frequencies and the circular piston source model was used for high frequencies.

Different behaviour at low and high frequencies is apparent from the simulation. At frequency of $100 \mathrm{~Hz}$ the wavelength is quite long $\left(3.43 \mathrm{~m}\right.$ at the temperature $\left.20^{\circ} \mathrm{C}\right)$, thus more than three times longer than the overall length of the simulated line array source $(1.05 \mathrm{~m})$. Therefore, the directional pattern is almost omnidirectional. Nevertheless, at the frequency of $6 \mathrm{kHz}$ the wavelength is only $0.057 \mathrm{~m}$ long, which is much less than the line source length. Due to that, there are dark blue areas in the simulation, where the individual sources are summing with similar or even the same amplitude, however, with the phase difference higher than $120^{\circ}$ and lower than $240^{\circ}$ [7]. The result is that the energy of the sources in this area (at this frequency) is cancelled instead summed. On the other hand, the bright colours denote the areas where the sources are in phase.

Anyway, the important feature of the simulated line array source is the front-back symmetry. Simulations are shown only for front directions in the figures presented in this paper, thus only the directional response in the front of the line source is displayed. That is because the measurements of the real directional characteristics were done only for this interval of angles.

\section{Directional characteristics measurement process}

Directional response data measured on real sources can provide more accurate information about some phenomena (such as diffraction) not considered in the simulations with ideal sources. A standard procedure of directional characteristic measurement is measuring the acoustic pressure effective value of loudspeaker fed with harmonic signal at given frequency [8]. A special turntable is employed to rotate the loudspeaker around its axis. However, this process is not very flexible when higher number of frequencies or the individual loudspeakers of composite sound source are needed to be measured. It is the reason why we designed and utilized the method named directional-frequency analysis.

\subsection{Directional-Frequency analysis}

The method is based on the use of a turntable with the constant angular speed of rotation and the broadband signal with con- stant power spectral density (e.g. white noise) feeding the measured loudspeaker. The output signal of a measurement microphone is recorded during rotation of the loudspeaker. The next step is timefrequency analysis of the recorded signal. For instance a discreet short-time Fourier transform (STFT) can be utilized for this purpose. It can be written as

$$
s_{S T F T}\left(e^{j \omega_{i}}, m\right)=\sum_{n=-\infty}^{\infty} s[n] w[n-m] e^{j \omega_{i}, n}
$$

where $s[n]$ is the sequence of recorded signal samples, $w[n]$ is the weighting function (Hamming window was used in our case), $\omega_{i}$ are frequency points, and $m$ are time points. Nonetheless, several other transformations could be used as well. Due to the known constant radial frequency of turntable rotation, the time points can be substituted by the corresponding angle points. The angle $\alpha$ between the acoustic axis of the loudspeaker and the line connecting the point of measurement and the loudspeaker reference point can be written as

$$
\alpha=v_{r} t[\mathrm{rad}]
$$

where $t$ is the time from the beginning of the record and $v_{r}$ is the radial frequency in $\mathrm{rad} \cdot \mathrm{s}^{-1}$. Following relation holds for time point $m$ and angle $\alpha$

$$
m=\alpha \frac{f_{s}}{v}
$$

where $f_{s}$ is the sample rate of recorded signal. Therefore, results of time-frequency analysis can be interpreted as the directional-frequency analysis results. Set of transformed coefficients provide frequency responses for the particular angles of rotation as well as directional characteristics for particular frequencies. Frequency characteristics are obtained for the points $\omega_{l}$ with $l=0,1, \ldots, N-1$ ( $N$ is the length of weighting window) and directional characteristics are obtained for the points $m=0,1, \ldots, 2 \pi f_{s} / v$. Both the frequency resolution $\Delta \omega$ and the angular resolution $\Delta \alpha$ depend on the length of weighting window $N$ according to equations

$$
\begin{aligned}
& \Delta \omega=\frac{f_{s}}{N} \\
& \Delta \alpha=\frac{N}{f_{s}} v
\end{aligned}
$$

\subsection{Process of measurement}

The measurement was done in the semi-anechoic chamber to simulate the free field. The critical frequency of the chamber is $200 \mathrm{~Hz}$. Because of the dimensions of the line array source it was easier to rotate the measurement microphone around the source instead of the rotation of the line array source itself. The turntable was utilized to rotate the microphone fixed on a $1.25 \mathrm{~m}$ long beam. The reference point of the microphone was set to the same height as the reference point of the measured speaker. The turntable allows setting of the lowest constant speed of rotation (convenient for the highest precision of analysis) to $\pi / 360 \mathrm{rad} \cdot \mathrm{s}^{-1}$. The directional 

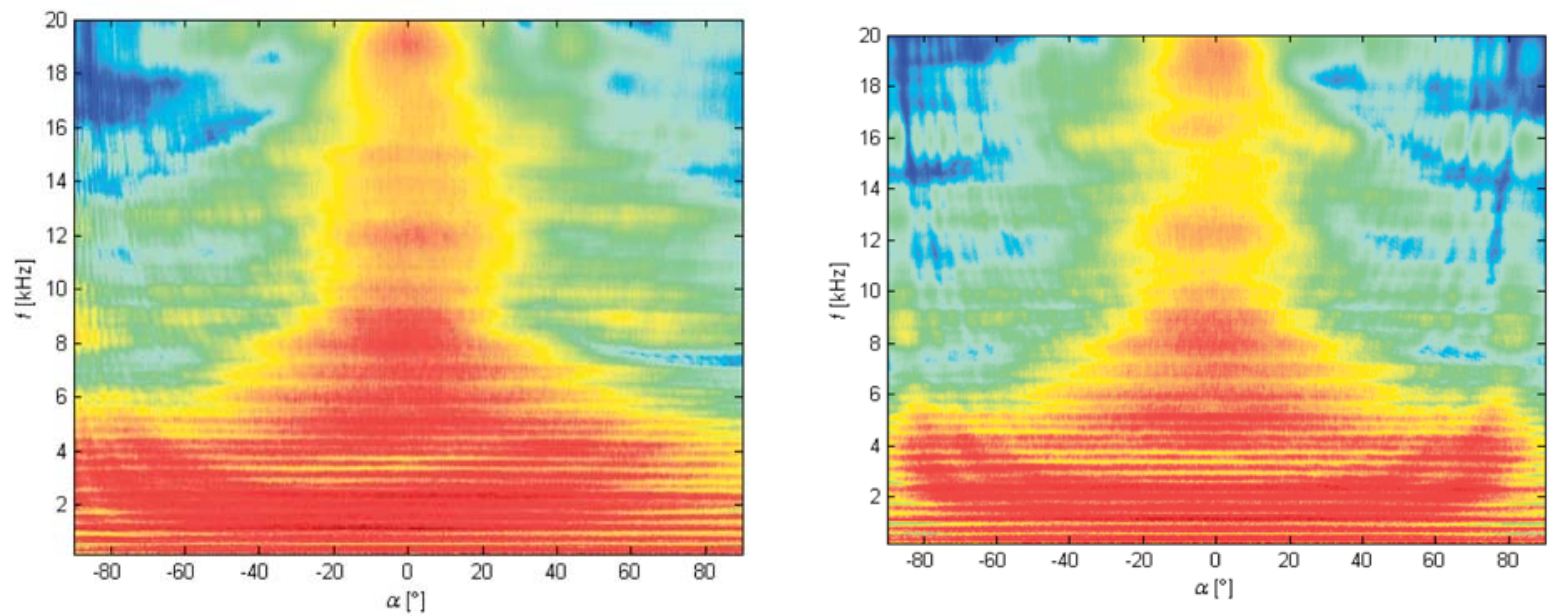

Fig. 3 The directional-frequency analysis of the first (left) and the fourth (right) individual source (from the front view and the left side)
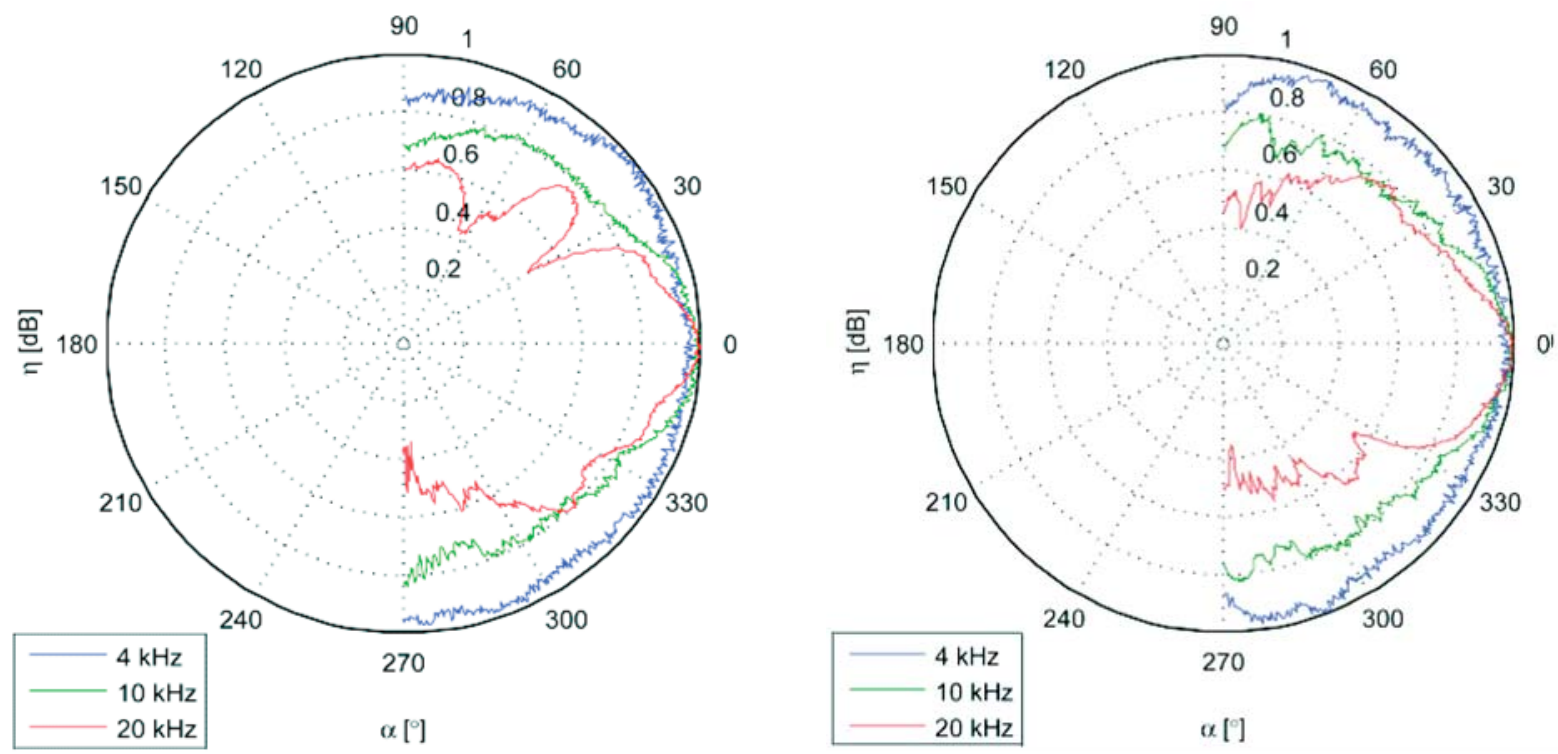

Fig. 4 Directional characteristics of the first (left) and the fourth (right) individual source (from the front view and the left side)

characteristics were measured only for the angles from the inter$\operatorname{val}\left(-90^{\circ} ; 90^{\circ}\right)$. White noise with a crest factor of 1.5 was used as a measurement signal. Sample rate was set to $48 \mathrm{kHz}$.

\subsection{Measurement results}

The results of the measurement and analysis can be seen in Figs. 3 and 4. Floating average filter with the width of $1 / 6$ octave was used to smooth the resulting characteristics [9]. There is a comparison of the outer and the middle segment of the line array source in Fig. 3.

The differences are probably the consequence of different position within the line array source, thus the different diffraction on the edges of the line array source enclosures. Another feature of the sources visible in the analysis is that their directional pattern gets narrower at the frequency about $6 \mathrm{kHz}$. Different behaviour of the line array source segments can also be seen in Fig. 4. The differences are the most apparent at the highest frequencies.

\section{Real sound source simulation}

Because of the different directional behaviour of the real sources beside the ideal ones, the resulting coverage pattern of the area in front of the line array source is also different. The phase relations between the sources (at the certain point) remain unchanged, though the amplitude ratios between the sources are modified by the individual directional characteristics. Therefore, the measured 
directional data should be implemented into the simulations with ideal sound sources.

At the certain point of the space it can be done by multiplying the ideal source amplitude by the normalized value of the directional characteristic at given frequency. For the $i$-th ideal point source with applied real source directionality the velocity potential at the certain point $\mathrm{X}$ of the space can be calculated using (3) as

$$
\Phi_{i}=\frac{A_{i} d_{i}(f, \alpha) e^{-j k r_{i}}}{r_{i}}
$$

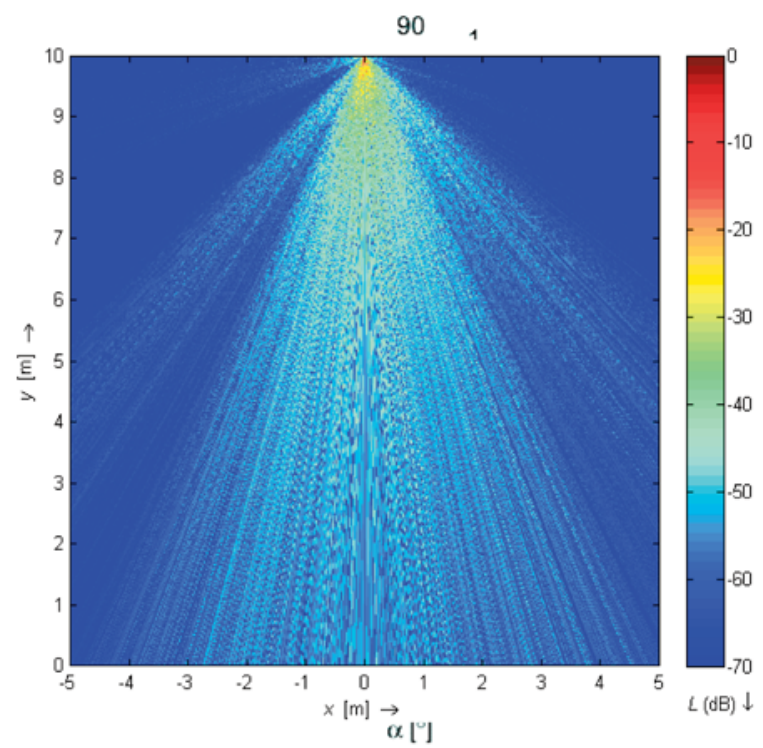

a)

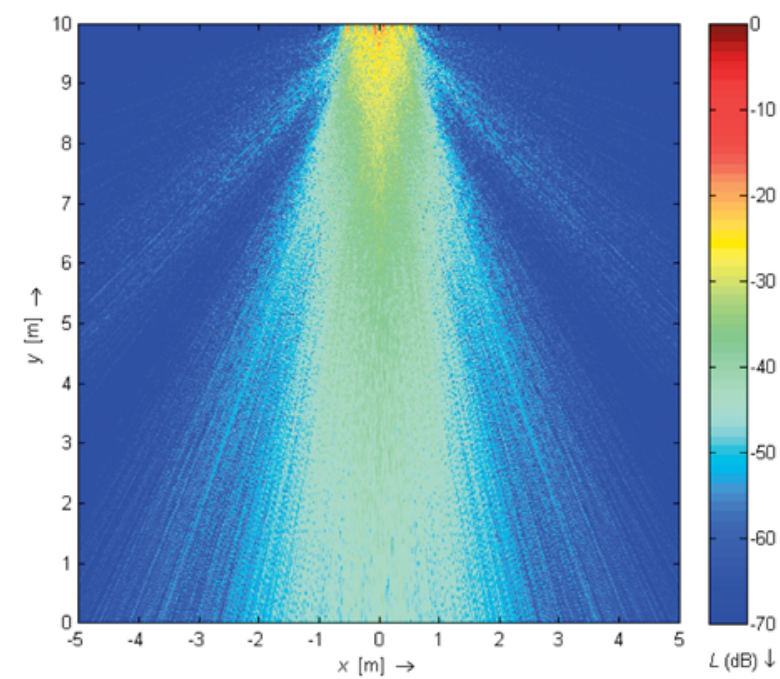

c) where $d_{i}\left(d_{i} \in(0,1)\right)$ is the normalized directional characteristic coefficient corresponding to the given simulated frequency $f$ of the source and the angle $\alpha$ between the acoustic axis of the loudspeaker and the line connecting the speaker reference point and calculated point $\mathrm{X}$. The accuracy of the correct coefficient determination depends on the mentioned resolution of the directionalfrequency analysis.

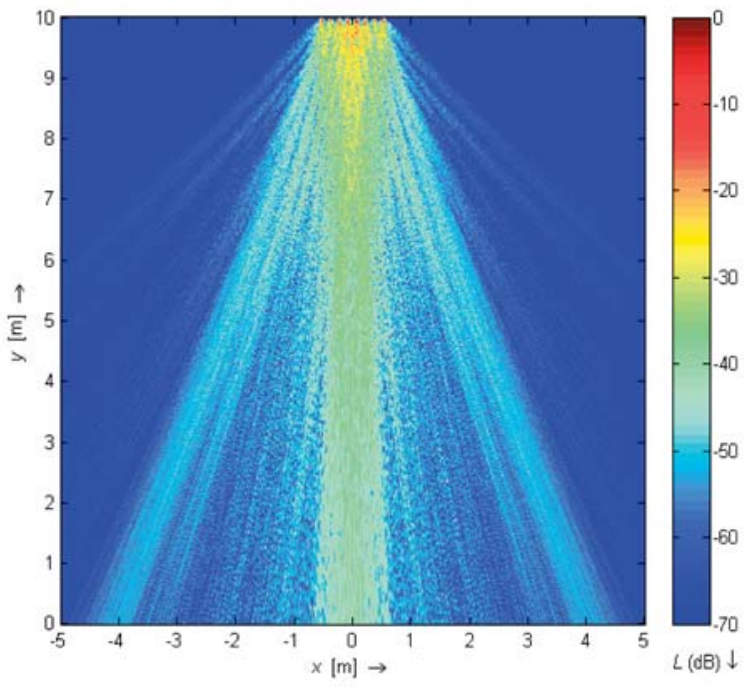

b)

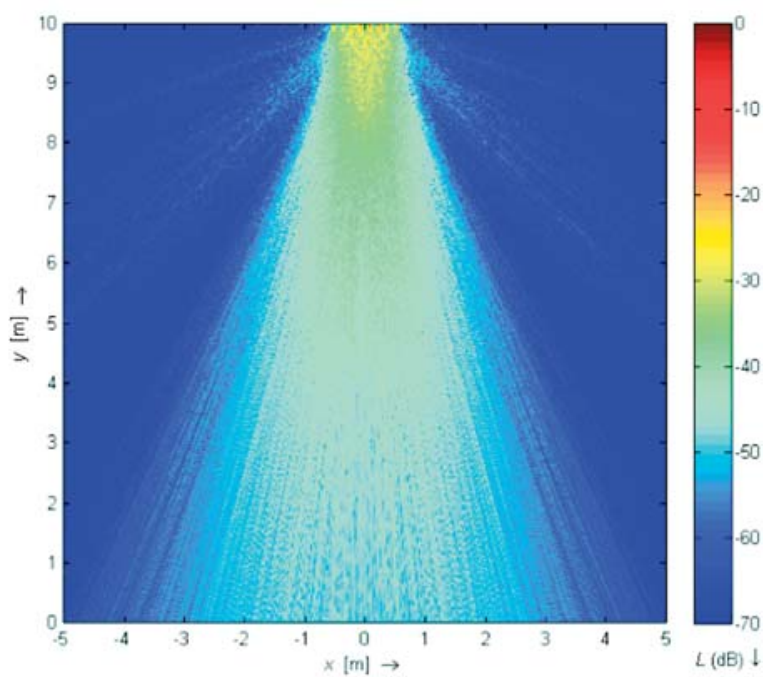

d)

Fig. 5 The simulation of the single source with applied directional characteristic at frequency of $4 \mathrm{kHz}(\mathrm{a})$; The simulation of the line array source with applied directional characteristics at frequency of $6 \mathrm{kHz}(\mathrm{b}), 1 \mathrm{kHz}(\mathrm{c})$ and $500 \mathrm{~Hz}(\mathrm{~d})$ 
An example of the single source simulation with applied directional characteristic is in Fig. 5 (a). Compared with the ideal circular piston source (see Fig. 1) the source in Fig. 5 (a) has a narrower directional pattern (at the same frequency). Therefore, the directivity of the overall line array source will be higher as can be seen in Fig. 5 (b). However, the directional patterns of the individual sources are not as wide as the ideal ones, thus the ratios of the source amplitudes are higher at the places of sources cancellation (caused by the big phase difference), which means the cancelation is not such dramatic. And the acoustic pressure variance (at this frequency) is also lower. The width of the main beam (the area of the highest acoustic energy) depends on frequency, or on wavelength (higher frequency means narrower beam) and the overall length of the line array source (longer source means narrower beam) [10]. Figure 5 (c) shows the line array source at the frequency of $1 \mathrm{kHz}$. Its directional pattern is wider than in Fig. 5 (b) due to lower frequency. Fig. 5 (d) demonstrates the further directional pattern widening at the frequency of $500 \mathrm{~Hz}$.

Although, the main result of the simulation is the figure of predicted sound field, we can also compare the results of the simulation of the ideal models of sound sources, the simulation of sound sources with imported directional characteristics and the real measured line array source. Accordingly, the comparison of

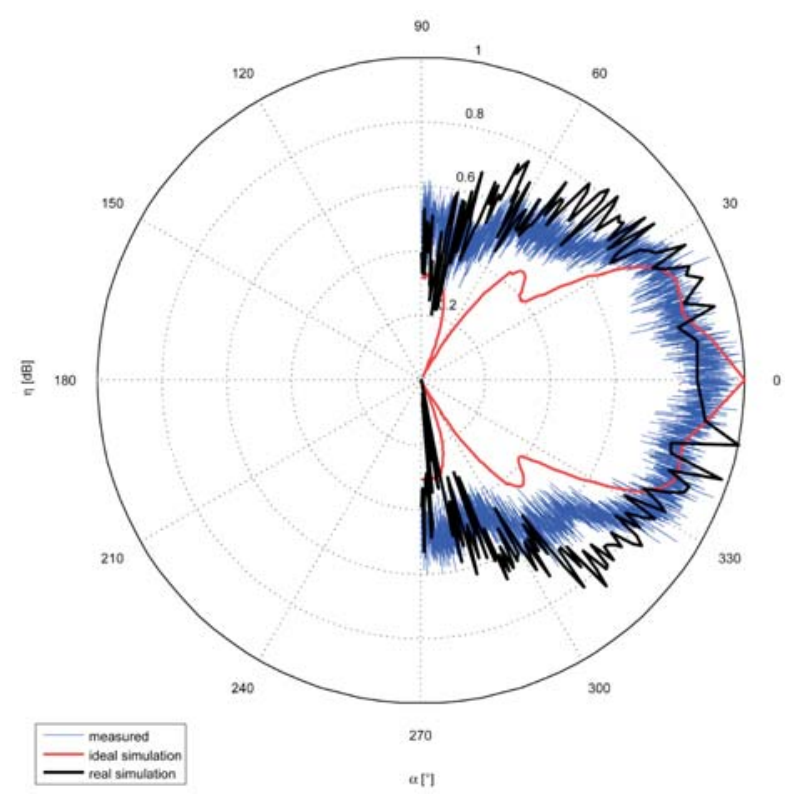

Fig. 6 The comparison of the measured and simulated directional characteristics of line array source containing eight speakers with spacing $0.15 \mathrm{~m}$ on $1 \mathrm{kHz}$ the directional characteristics in $1 \mathrm{~m}$ distance on axis and frequency $1 \mathrm{kHz}$, measured or simulated with line array sources containing eight segments with $0.15 \mathrm{~m}$ spacing is shown in Fig. 6.

The measured directional characteristic was smoothed with the floating average filter with the width of $1 / 3$. It can be seen that the trace of directional characteristic simulated with the sources with imported real characteristics (black line) is more similar to the measured characteristic (blue line) than the one simulated with the ideal models of sources (red line). However, the simulated characteristics were made with the resolution of $0.01 \mathrm{~m}$, which is not very sufficient especially around $0^{\circ}$, where the number of values is quite small. The higher degree of precision can be reached with even higher resolution, but the computational cost will substantially increase too.

\section{Conclusion}

The acoustic line array source simulation method was presented utilizing the directional-frequency analysis to implement the directional characteristics of the real measured acoustic sources. Presented method of loudspeaker measurement and directional-frequency analysis allows obtaining the data very quickly as compared with the standard measurement process. Directional characteristics can be obtained as the frequency slices of the directional-frequency analysis while frequency responses can be obtained as the time (angle) slices. Furthermore, various kinds of analysis can be applied on the measured signals and the frequency or directional resolution can be adjusted as necessary. The directional characteristics were implemented into the simulations and the differences between the ideal case and the real sources simulations were shown.

Resulting simulations are useful for the visualization of the acoustic pressure coverage of the area. The function created in Matlab is able to simulate individual sources as well as line array sources or the interaction of any other spatial configuration of multiple sources.

The only drawback is the big computational complexity when simulating higher number of sources with high resolution. The overall time of the simulation of eight sources and the resolution of $0.01 \mathrm{~m}$ on Intel Core i $72800 \mathrm{GHz}$ CPU and the RAM memory of $4 \mathrm{~GB}$ was approx. 14 minutes, while with ten times higher resolution $(0.001 \mathrm{~m})$ the computational time increased to approx. 99 minutes.

\section{Acknowledgement}

The described research was performed in laboratories supported by the SIX project; the registration number CZ.1.05/2.1.00/ 03.0072, the operational program Research and Development for Innovation. 


\section{COMMNICOIIIONS}

\section{References}

[1] THEILE, G., WITTEK, H.: Wave Field Synthesis - A Promising Spatial Audio Rendering Concept, to be published in J. of Image Information and Television Engineers, 2007, pp. 77. Online: http://www.irt.de/fileadmin/media/downloads/Produktion/ WaveFieldSynthesis.pdf

[2] RUND, F.: Simulace zvukoveho pole vice zdroju [Simulation of the Sound Field of Multiple Sources]. Proc. of Technical Computing Prague, Praha : Humusoft, 2009, pp. 5. ISBN 978-80-7080-733-0

[3] BRUNEAU, M.: Fundamentals of Acoustics. London : ISTE Ltd, 2006. ISBN-13: 978-1-905209-25-5

[4] SKVOR, Z.: Akustika a elektroakustika [Acoustics and Electroacoustics]. Praha, Academia, 2001, pp. 527. ISBN 80-200-0464-0

[5] RUZICKOVA, M.: Discrete and Differential Equations in Applied Mathematics. Published in Communications - Scientific Letters of the University of Zilina, No. 2, 2012. ISSN 1335-4205.

[6] RAICHEL, D. R.: The Science and Application of Acoustics. New York: Springer, 2006. ISBN-13: 978-0387-26062-4

[7] OLSON, H. F.: Elements of Acoustical Engineering. New York: D. Van Nostrand Company, Inc., 1940, pp. 368.

[8] MERHAUT, J. et al: Prirucka elektroakustiky [The Electroacoustic Handbook]. Praha, Statni nakladatelstvi technicke literatury, 1964, pp. 610.

[9] MALACKA, Z.: Pursuit Curves and Ordinary Differential Equations. Published in Communications - Scientific Letters of the University of Zilina, No. 1, 2012, ISSN 1335-4205.

[10] ZIOMEK, L. J.: Foundamentals of Acoustic Field Theory and Space-Time Signal Processing. Boca Raton : CRC Press, 1995. ISBN: 0-8493-9455-4. 